Journal Club

Editor's Note: These short, critical reviews of recent papers in the Journal, written exclusively by graduate students or postdoctoral fellows, are intended to summarize the important findings of the paper and provide additional insight and commentary. For more information on the format and purpose of the Journal Club, please see http://www.jneurosci.org/misc/ifa_features.shtml.

\title{
Lactate Transport and Metabolism in the Human Brain: Implications for the Astrocyte-Neuron Lactate Shuttle Hypothesis
}

\author{
Chase R. Figley \\ Departments of Neuroscience and Psychological and Brain Sciences, Johns Hopkins University, Baltimore, Maryland 21218, and The F.M. Kirby Research \\ Center for Functional Brain Imaging, Kennedy Krieger Institute, Baltimore, Maryland, 21205 \\ Review of Boumezbeur et al.
}

Lactate is the metabolic byproduct of glycolysis, a process that, despite being less efficient than the complete oxidation of glucose to carbon dioxide and water, occurs throughout the brain. Glycolytic metabolism increases dramatically in response to ischemic events, but even during periods of sufficient oxygen availability, so-called "aerobic glycolysis" may account for as much as $10-12 \%$ of glucose metabolism in the adult human brain (Vaishnavi et al., 2010). Moreover, in addition to resting-state glycolysis and baseline lactate production, brain activity has been shown to significantly increase local lactate concentrations (for review, see Mangia et al., 2009).

It is thought that glycolysis is invoked to quickly, albeit inefficiently, provide energy; and because lactate has been implicated in the regulation of cerebral microcirculation (causing arteriole dilation), glycolytic increases might also play important roles in redirecting blood flow to oxygen-deprived brain regions during acute trauma and regulating cerebral blood flow

Received Dec. 17, 2010; revised Jan. 12, 2011; accepted Jan. 16, 2011.

This work was supported by a Canadian Institutes of Health Research Postdoctoral Fellowship Award. I thank Dr. Fawzi Boumezbeur for supplying his original data, as well as Teresa McAdam for proofreading and critiquing preliminary drafts of the manuscript.

Correspondence should be addressed to Chase R. Figley, Room 110 Ames Hall, Johns Hopkins University, Baltimore, MD 21218, E-mail: cfigley@jhu.edu.

DOI:10.1523/JNEUROSCI.6612-10.2011

Copyright $\odot 2011$ the authors $\quad 0270-6474 / 11 / 314768-03 \$ 15.00 / 0$ in step with local metabolism under normal physiological conditions (Gordon et al., 2008). Although it is now mostly agreed that activity-dependent lactate increases occur, and that these are important for cerebrovascular coupling and the production of functional neuroimaging signals (Figley and Stroman, 2011), both the cellular origin and the ultimate fate of lactate remain to be definitively confirmed.

In a recent report, Boumezbeur et al. (2010) demonstrated that intravenous infusions of ${ }^{13} \mathrm{C}$-labeled lactate, combined with in vivo ${ }^{13} \mathrm{C}$ magnetic resonance spectroscopy (MRS), can be used to assess lactate transport kinetics and the cerebral metabolic rate of blood-borne (plasma) lactate in the human cerebral cortex, and to quantify the absolute and relative amounts of plasma lactate that are metabolized by neurons and glia. Based on a reversible Michaelis-Menton model, they showed that the rate of unidirectional lactate transport from the blood to the brain $\left(V_{\text {in }}\right)$, the brain lactate concentration $\left([\mathrm{Lac}]_{\mathrm{B}}\right)$, and the cerebral metabolic rate of lactate (CMRlac) increased approximately linearly with plasma lactate concentration $\left([\mathrm{Lac}]_{\mathrm{P}}\right)$.

Boumezbeur et al. (2010) also compared the fractional enrichments of $\left[4-{ }^{13} \mathrm{C}\right]$ glutamine and $\left[4-{ }^{13} \mathrm{C}\right]$ glutamate and found that the ratio of labeled glutamine/ glutamate was similar $(0.78 \pm 0.04$ and $0.87 \pm 0.04$, respectively) after $2 \mathrm{~h}$ of in- fusion witheither $\left[3-{ }^{13} \mathrm{C}\right]$ lactate or $\left[1-{ }^{13} \mathrm{C}\right]$ glucose, respectively. These findings, along with the lactate flux through the tricarboxylic acid (TCA) cycle, which they estimated as $0.50 \pm 0.02 \mu \mathrm{mol} / \mathrm{g} / \mathrm{min}$ in neurons and $0.15 \pm 0.02 \mu \mathrm{mol} / \mathrm{g} / \mathrm{min}$ in glia, suggest that neurons metabolized $\sim 80 \%$ of the radiolabeled lactate (i.e., approximately four times more than glia). In addition, their results indicate that plasma lactate might play a significant role in supporting oxidative brain metabolism. They proposed that, under extreme $[\mathrm{Lac}]_{\mathrm{P}}$ conditions, the theoretical limit of lactate transport $\left(V_{\max }\right)$ would allow plasma lactate to fuel up to $60 \%$ of all cerebral metabolism and TCA cycle activity, and that the overall contribution could be as high as $10 \%$, even under normal physiological conditions (i.e., where $[\mathrm{Lac}]_{\mathrm{P}} \approx 1.0 \mathrm{mmol} / \mathrm{L}$ ).

These data are especially interesting within the context of other recent reports, and might help to clarify certain debates regarding neurometabolism and cerebrovascular coupling mechanisms. Thus, I would like to briefly expand on Boumezbeur et al.'s (2010) initial discussion in a few key areas.

\section{Contribution of plasma lactate to} brain metabolism

Boumezbeur et al. (2010) found that both $[\mathrm{Lac}]_{\mathrm{B}}$ and CMRlac were linearly related to $[\mathrm{Lac}]_{\mathrm{P}}$, such that $[\mathrm{Lac}]_{\mathrm{B}}=0.63[\mathrm{Lac}]_{\mathrm{P}}$ with an $R^{2}=0.75$, and CMRlac $=$ 


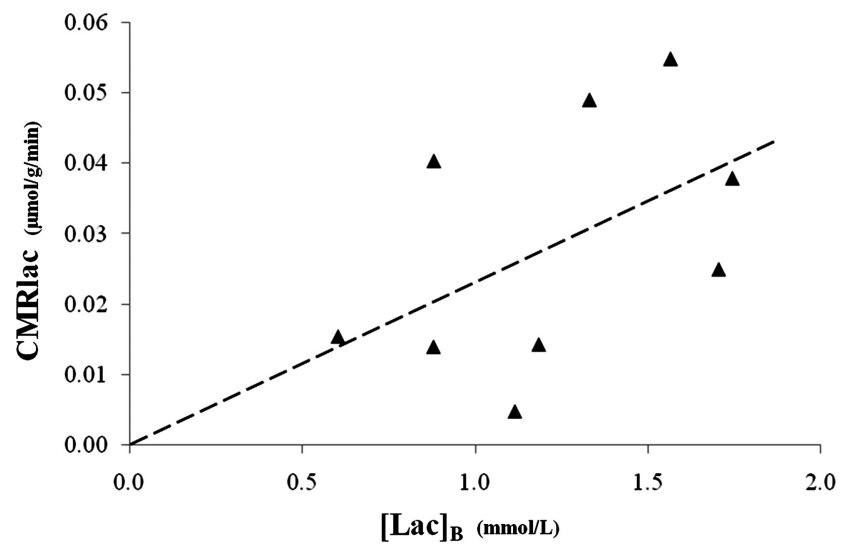

Figure 1. The cerebral metabolic rate of plasma-borne lactate as a function of brain lactate concentration. The dashed line represents the least-squares linear regression CMRlac $=0.023[\mathrm{Lac}]_{B}$, which yields an $R^{2}=0.23$, or 0.40 by including a point at the origin. Data were generously supplied by Boumezbeur et al. (2010).

$0.019[\mathrm{Lac}]_{\mathrm{P}}-0.007$ with an $R^{2}=0.71$ (Boumezbeur et al., 2010, their Fig. 6b,c). This means that $\sim 75 \%$ of the $[\mathrm{Lac}]_{\mathrm{B}}$ and $71 \%$ of the CMRlac changes were related to $[\mathrm{Lac}]_{\mathrm{P}}$. Interestingly, the correlation between $[\mathrm{Lac}]_{\mathrm{B}}$ and CMRlac (which was not plotted by Boumezbeur et al.) was much less reliable: $\mathrm{CMRlac}=0.023[\mathrm{Lac}]_{\mathrm{B}}$ with an $R^{2}=0.23$ or 0.40 , if a data point was included at the origin (Fig. 1).

Given the high coefficients of determination between $[\mathrm{Lac}]_{\mathrm{P}}$ versus $[\mathrm{Lac}]_{\mathrm{B}}$ and $[\mathrm{Lac}]_{\mathrm{P}}$ versus CMRlac, it is perhaps surprising that there was such a poor correlation between $[\mathrm{Lac}]_{\mathrm{B}}$ and CMRlac. Because this regression was based on only nine or 10 data points, it is possible that noise and/or a small number of outliers may have reduced the overall fit. However, other recent experiments support the notion that there are also sources of structured variance between the measured $[\mathrm{Lac}]_{\mathrm{B}}$ and CMRlac (discussed below).

\section{Cellular compartmentalization of lactate metabolism}

Although the overall TCA cycle rate reported by Boumezbeur et al. (2010) $(\sim 0.65$ $\mu \mathrm{mol} / \mathrm{g} / \mathrm{min}$ ) was within the range of previously reported values for humans, the TCA flux though the neuronal pool $(0.50 \mu \mathrm{mol} /$ $\mathrm{g} / \mathrm{min}$ ) was unusually low, and the flux through the glial pool $(0.15 \mu \mathrm{mol} / \mathrm{g} / \mathrm{min})$ was quite high (Hyder et al., 2006). The authors postulated that this might have resulted from the high proportion of white matter $(\sim 53 \%)$ in their volume of interest; a justification that is supported by (1) previous MRS studies (which also showed lower TCA fluxes in white matter), (2) the fact that white matter contains more glia than gray matter (Azevedo et al., 2009), and (3) the finding that glia have lower TCA cycling rates than neurons (Hyder et al., 2006; Boumezbeur et al., 2010).

However, by uncovering large variations in the amount of aerobic glycolysis occurring throughout the resting human brain, another recent study (Vaishnavi et al., 2010) offers a second possible explanation for the low neuronal TCA flux. Of particular interest, these authors found extremely high rates of glycolysis throughout the medial and lateral parietal lobes (including the posterior cingulate/precuneus), corresponding to the parietal-occipital volumes examined by Boumezbeur et al. (2010). Because the fractional enrichment of plasma glucose was found to be negligible (Boumezbeur et al., 2010, their Fig. $2 d$ ), even high rates of regional aerobic glycolysis would produce only trace amounts of labeled lactate. Therefore, mixing between the unlabeled lactate pool (from regional brain glycolysis) and the labeled lactate pool (transported from the blood) would lead to the incorporation of unlabeled carbons in the downstream glutamate and glutamine pools, and a systematic underestimation of the TCA cycle rates.

\section{Relevance to the astrocyte-to-neuron lactate shuttle}

The studies presented here support the idea that lactate is used as a significant source of fuel for brain metabolism, and that it likely originates from two sources: locally (from aerobic glycolysis) and distally (via the blood). A controversial theory encompassing the production and consumption of cerebral lactate has come to be known as the astrocyte-neuron lactate shuttle hypothesis (ANLSH). In its most basic form, the ANLSH posits that lactate, synthesized by astrocytes, is consumed by neurons as a metabolic substrate (Pellerin et al., 2007). Although the
Boumezbeur et al. (2010) study does not address the production of lactate, it firmly supports the proposition that lactate (even from the bloodstream) is taken up and used by neurons under normal physiological conditions.

Several studies using in vivo MRS (Mangia et al., 2009) and microdialysis (Caesar et al., 2008) have shown that lactate levels reliably increase with the onset of brain activation. This implies that regional glycolysis exceeds lactate metabolism and clearance during the initial phase of activation. However, at some point during sustained activity, the rates of lactate production and elimination appear to achieve a new elevated steady state, which lasts until brain activation, and then lactate levels, return to baseline (Caesar et al., 2008). In such cases, there are two outstanding questions: (1) what is the nature of the lactate flux (i.e., turnover/exchange from blood to cells, from one cell type to another, or from cells to blood), and (2) how do the elevated lactate concentrations return to baseline (i.e., removal in the blood vs local metabolism)?

Recent data from rat brain slices suggest that astrocytes have a twofold to fourfold higher capacity for lactate uptake and clearance (via astrocyte syncytia) compared to direct neuronal uprake or shuttling of lactate from astrocytes to neurons (Gandhi et al., 2009), implying that astrocytes take up, disperse, and remove lactate by releasing it to the blood or perivascular fluid. However, the results of Boumezbeur et al. (2010) suggest that this may not be the case in the living human brain. Their in vivo results demonstrate that, especially during elevated $[\mathrm{Lac}]_{\mathrm{p}}$, there is a net influx of lactate from the blood to the brain, and that this lactate, although metabolized by both cell types, is primarily consumed by neurons. Therefore, along with the high affinity for astrocytic lactate uptake (Gandhi et al., 2009) and the fact that astrocytes surround $99 \%$ of the total cerebrovascular surface area (providing more direct access to plasma lactate), these findings suggest that astrocytes might have played a large role in shuttling lactate toward the neurons.

Finally, although the ANLSH predicts that neurons oxidize lactate, it also acknowledges that they may consume significant amounts of glucose (Pellerin et al., 2007). Notwithstanding that, the prediction that plasma lactate could supply up to $60 \%$ of the brain's oxidative fuel, combined with reports that lactate administration is sufficient to support brain function during hypoglycemia (Boumez- 
beur et al., 2010, and references therein), implies that neurons are capable of transporting and metabolizing large quantities of lactate in vivo: findings that are consistent with previous in vitro spectroscopy studies showing that cultured neurons might preferentially oxidize lactate as their primary metabolic substrate (for review, see Figley and Stroman, 2011). Therefore, despite a higher nominal affinity for lactate uptake into astrocytes (Gandhi et al., 2009), empirical data suggest that large amounts of lactate are transported into neurons, and that this is not likely to represent a rate-limiting step for the ANLSH.

\section{References}

Azevedo FA, Carvalho LR, Grinberg LT, Farfel JM, Ferretti RE, Leite RE, Jacob Filho W, Lent R, Herculano-Houzel S (2009) Equal numbers of neuronal and nonneuronal cells make the human brain an isometrically scaled-up primate brain. J Comp Neurol 513:532-541.

Boumezbeur F, Petersen KF, Cline GW, Mason GF, Behar KL, Shulman GI, Rothman DL (2010) The contribution of blood lactate to brain energy metabolism in humans measured by $d y-$ namic ${ }^{13} \mathrm{C}$ nuclear magnetic resonance spectroscopy. J Neurosci 30:13983-13991.

Caesar K, Hashemi P, Douhou A, Bonvento G, Boutelle MG, Walls AB, Lauritzen M (2008) Glutamate receptor-dependent increments in lactate, glucose and oxygen metabolism evoked in rat cerebellum in vivo. J Physiol 586:1337-1349.

Figley CR, Stroman PW (2011) The role(s) of astrocytes and astrocyte activity in neurometabolism, neurovascular coupling, and the production of functional neuroimaging signals. Eur J Neurosci 33:577-588.

Gandhi GK, Cruz NF, Ball KK, Dienel GA (2009) Astrocytes are poised for lactate trafficking and release from activated brain and for supply of glucose to neurons. J Neurochem 111:522-536.

Gordon GR, Choi HB, Rungta RL, Ellis-Davies
GC, MacVicar BA (2008) Brain metabolism dictates the polarity of astrocyte control over arterioles. Nature 456:745-749.

Hyder F, Patel AB, Gjedde A, Rothman DL, Behar KL, Shulman RG (2006) Neuronalglial glucose oxidation and glutamatergicGABAergic function. J Cereb Blood Flow Metab 26:865-877.

Mangia S, Giove F, Tkác I, Logothetis NK, Henry PG, Olman CA, Maraviglia B, Di Salle F, Uğurbil K (2009) Metabolic and hemodynamic events after changes in neuronal activity: current hypotheses, theoretical predictions and in vivo NMR experimental findings. J Cereb Blood Flow Metab 29:441-463.

Pellerin L, Bouzier-Sore AK, Aubert A, Serres S, Merle M, Costalat R, Magistretti PJ (2007) Activity-dependent regulation of energy metabolism by astrocytes: an update. Glia 55:1251-1262.

Vaishnavi SN, Vlassenko AG, Rundle MM, Snyder AZ, Mintun MA, Raichle ME (2010) Regional aerobic glycolysis in the human brain. Proc Natl Acad Sci U S A 107:1775717762. 